\title{
High Efficiency Waveguide-based Optical Amplifiers and Lasers Fabricated by Femtosecond-laser Induced Ion Migration
}

\author{
J. Hoyo ${ }^{1}$, P. Moreno-Zárate ${ }^{2}$, G. Escalante ${ }^{3}$, J.A. Vallés ${ }^{4}$, P. Fernández ${ }^{3}$, J. Solis ${ }^{*}$ \\ 1. Laser Processing Group, Instituto de Óptica, CSIC, Serrano 121, 28006 Madrid, Spain \\ 2. Industrial Engineering, Tepexi Higher Technological Institute, Av. Tecnológico S/N, 74690, Tepexi de Rodríguez, Mexico \\ 3. Depto. de Física de Materiales, Facultad de Físicas, Univ.Complutense, 28040 Madrid, Spain \\ 4. Departamento de Física Aplicada-I3A, Facultad de Ciencias, Universidad de Zaragoza, Zaragoza 50009, Spain \\ *e-mail: j.solis@io.cfmac.csic.es
}

The modification of the glass composition by high repetition rate femtosecond ( $\mathrm{fs}$ ) laser pulse irradiation appears as a very attractive method for the production of photonic devices [1]. It has recently been shown that this mechanism enables producing refractive index changes, $\Delta \mathrm{n}>10^{-2}$, leading to the production of highly efficient, and controllable passive waveguides in $\mathrm{P}_{2} \mathrm{O}_{3}-\mathrm{La}_{2} \mathrm{O}_{3}-\mathrm{K}_{2} \mathrm{O}$-based glasses [2]. The index contrast $(\Delta \mathrm{n})$ is caused by the enrichment of $\mathrm{La}_{2} \mathrm{O}_{3}$ in the guiding region which is depleted in $\mathrm{K}_{2} \mathrm{O}$ due to cross migration of both elements. Still, it has also been shown [3] that the guiding region is enriched in other lanthanides present in the glass (i.e. Er, Yb). This effect may be detrimental for the production of active devices. In this work we show that fs-laser induced ion-migration can similarly be used to produce high performance waveguide amplifiers and lasers. In fact, we show the feasibility of producing waveguides with different gain in the same glass sample by varying the writing parameters, increasing the flexibility of this technique in integrated photonics applications.

Femtosecond-laser writing, optical and structural characterization, and gain modeling details are described in Refs.[2-3]. The sample consists of a phosphate glass with $\sim 10$ mol.\% of $\mathrm{La}_{2} \mathrm{O}_{3}$ and $\mathrm{K}_{2} \mathrm{O}$, doped with 2 and 4 wt.\% of $\mathrm{Er}_{2} \mathrm{O}_{3}$ and $\mathrm{Yb}_{2} \mathrm{O}_{3}$, respectively. This sample was irradiated with a $400 \mathrm{fs}$ pulsed laser $(500 \mathrm{kHz}$ rep. rate), slit-shaped beam, focused $100 \mu \mathrm{m}$ underneath the surface. Sample scan speeds in the 40-60 $\mu \mathrm{m} / \mathrm{s}$ were used. After polishing the end facets of the waveguides, they were used to configure optical amplifiers and lasers whose performance was numerically simulated.

Figure 1 (left) shows the net gain achieved as a function of the waveguide length upon bidirectional pumping of two waveguides produced in the same glass sample with different writing parameters. The guiding region is enriched in $\mathrm{Er}^{3+}$ by $20 \%(\mathrm{Wg} 1)$ and $47 \%(\mathrm{Wg} 2)$. The right plot shows the slope efficiency of lasers produced using the same waveguides. A slope efficiency of $38 \%$ was achieved in $\mathrm{Wg} 1$ which could be bring up $42 \%$ by further optimization of the cavity configuration, a value comparable to the best ones achieved by more conventional waveguide production techniques [4].

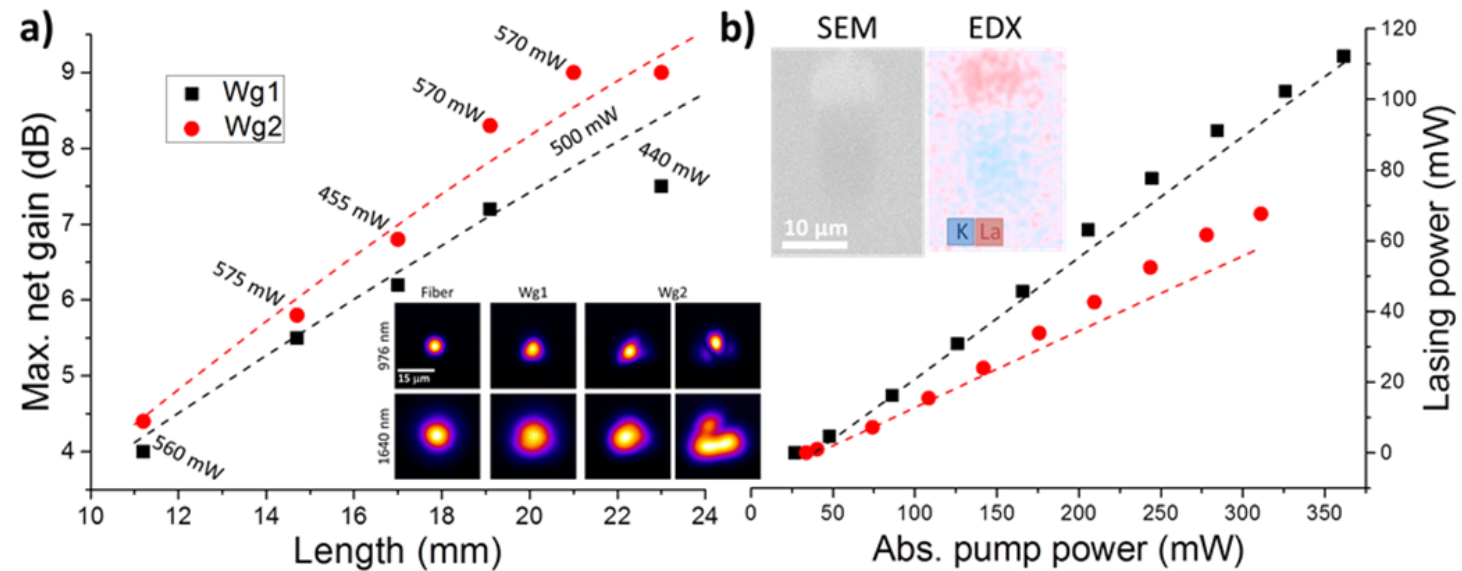

Fig. 1 (a) Net gain at $1534 \mathrm{~nm}$ of two waveguides (Wg1 and Wg2) written in the same glass sample as function of the waveguide length upon bidirectional pumping at $976 \mathrm{~nm}$. The pump power is indicated in the figure. Dashed lines correspond to the numerical simulations. The inset shows the fiber and waveguide guided modes, showing the multimodal behaviour of Wg2. (b) Laser slope efficiency of two lasers build-up using $\mathrm{Wg} 1$ and $\mathrm{Wg} 2$ as active medium and two fibre Bragg gratings $(99 \%$ and $69 \%$ reflectivity, $1 \mathrm{~nm}$ of bandwidth centered at $1535 \mathrm{~nm}$ ). The inset shows a SEM image and an EDX compositional map of Wg2. The waveguides were produced with a sample scanning speed of $60 \mu \mathrm{m} / \mathrm{s}, 1.4 \mathrm{~mm}$ slit width and $580 \mathrm{~nJ}$ laser pulses (Wg1) or $1.2 \mathrm{~mm}$ slit-width and $760 \mathrm{~nJ}$ pulses (Wg2), respectively.

\section{References}

[1] Y. Liu, M. Shimizu, B. Zhu, Y. Dai, B. Qian, J. Qiu, Y. Shimotsuma, K. Miura, and K. Hirao, "Micromodification of element distribution in glass using femtosecond laser irradiation.," Opt. Lett. 34, 136 (2009).

[2] T. Toney Fernandez, P. Haro-González, B. Sotillo, M. Hernandez, D. Jaque, P. Fernandez, C. Domingo, J. Siegel, and J. Solis, "Ion migration assisted inscription of high refractive index contrast waveguides by femtosecond laser pulses in phosphate glass.," Opt. Lett. 38, 5248 (2013) / J. Del Hoyo, R. M. Vazquez, B. Sotillo, T. T. Fernandez, J. Siegel, P. Fernández, R. Osellame, and J. Solis, "Control of waveguide properties by tuning femtosecond laser induced compositional changes," Appl. Phys. Lett. 105, 131101 (2014).

[3] T. T. Fernandez, B. Sotillo, J. del Hoyo, J.-A. Valles, R. M. Vazquez, P. Fernandez, and J. Solis, "Dual Regimes of Ion Migration in High Repetition Rate Femtosecond Laser Inscribed Waveguides," IEEE Photonics Technol. Lett. 27, 1068 (2015).

[4] G. Della Valle, a Festa, G. Sorbello, K. Ennser, C. Cassagnetes, D. Barbier, and S. Taccheo, "Single-mode and high power waveguide lasers fabricated by ion-exchange.," Opt. Express 16, 12334 (2008). 